\title{
CHARGE SINGULARITY AT THE VERTEX OF A SLENDER CONE OF GENERAL CROSS-SECTION
}

\author{
J. A. MORRISON
}

Abstract. Let the equation of the cone be given in spherical coordinates $(r, \theta, \varphi)$ by $f\left(\epsilon^{-1} \tan \theta \cos \varphi, \epsilon^{-1} \tan \theta \sin \varphi\right)=0$, with $\theta=$ $O(\epsilon)$, where $0<\epsilon \ll 1$ is a parameter. The potential $V$ satisfies Laplace's equation $\nabla^{2} V=0$ exterior to the cone, with boundary condition $V=0$ on the cone. A solution is sought in the form $V=$ $r^{\rho} U(\theta, \varphi)$, and the boundary condition leads to an eigenvalue problem for $\rho$. The quantity of interest is the smallest $\rho>0$. For $0<\rho<1$ there is a singularity in the surface charge density of the form $r^{\rho-1}$ at the vertex of the cone, in addition to any edge singularities that may be present.

Near the cone, for $\boldsymbol{\theta}=\mathrm{O}(\boldsymbol{\epsilon})$, the complex variable $\tau=\epsilon^{-1} \tan (\theta / 2) e^{i \varphi}$ is introduced, and in the inner limit the equation for $U$ tends to the 2 -dimensional Laplace equation as $\boldsymbol{\epsilon} \rightarrow 0$. The eigenvalue problem is solved by a singular perturbation procedure, by matching the inner solution to the outer solution, away from the cone. Let $C_{\epsilon}$ denote the contour in the $\tau$-plane which corresponds to the cone. Suppose that the domain exterior to the circle $|w|=\lambda(\epsilon)$ is mapped conformally onto the domain exterior to $C_{\epsilon}$ by $\tau=g(w, \epsilon)$, with $\tau-w-\alpha_{0} \rightarrow 0$ as $|w| \rightarrow \infty$, so that $\dot{\lambda}(\epsilon)$ is the outer radius of $C_{\epsilon}$. It is found that

$$
\begin{aligned}
1 / 2 \psi(-\rho)+ & 1 / 2 \psi(1+\rho)-\psi(1)+\epsilon^{2} \kappa \rho(\rho+1) \\
& \sim \log (1 / \epsilon \lambda)+\epsilon^{2} \alpha_{0} \alpha_{0}^{*}
\end{aligned}
$$

where $\psi$ denotes the logarithmic derivative of the gamma function, the asterisk denotes complex conjugate, and $\boldsymbol{\kappa}$ is determined in terms of the mapping function $g(w, \epsilon)$. Details of the derivation of the above result will be given elsewhere [1]. Examples of cones with particular cross-sections have been considered.

The charge singularity at the vertex of a sectorial flat plate of angle $\chi$ has been investigated earlier by Morrison and Lewis [2]. For that problem, Laplace's equation was solved by separation of variables in conical coordinates. This leads to a dual eigenvalue problem for $\rho$ and for a separation constant $\delta$. Analytic expressions for the singularity strength were derived for $0<(2 \pi-\chi) \ll 1$, for $|\pi-\chi| \ll 1$, and for $0<\chi \ll 1$, by means of singular perturbation techniques, and 
numerical results were obtained for other values of $\chi$. The present result generalizes the one for small angles to cones of general crosssection.

References to related work are given in [1] and [2].

\section{REFERENCES}

1. J. A. Morrison, Charge singularity at the vertex of a slender cone of general cross-section, submitted to SIAM J. Appl. Math.

2. J. A. Morrison and J. A. Lewis, Charge singularity at the corner of a flat plate, SIAM J. Appl. Math., to appear.

Bell Laboratories, Murray Hill, New Jersey 07974 